\title{
PERIOD AND PERIOD CHANGES OF \\ RR LYRAE VARIABLES IN M15
}

\author{
A. J. WESSELINK \\ Yale University, U.S.A.
}

\begin{abstract}
New observations of the variables in M15 have been made with the Yale 40 -in. reflector for the study of periods and period changes.

Since the counting of cycles from the time of Bailey's observations to the present day cannot always be made unambiguously, there is a corresponding uncertainty in the nature and amount of the period changes. Good lightcurves are obtained even when the periods used for their reduction are incorrect because the number of cycles in a day, or in a month, or in a year, is wrong by one. When this happens, however, period changes are likely to be indicated, and these will be spurious.

Nevertheless, it can be shown that the periods of most variables have not been constant during the interval 1900-1970.

The situation could be improved if a relatively small number of plates were to be obtained each year for several decades, preferably with the same instrument. Individual accuracy could be improved by the use of the electronic camera.

The full contents of this paper will be published elsewhere.
\end{abstract}

\section{DISCUSSION}

Feast: What is the effect of variations in the light curve and shifting of time of maximum light?

Coutts: When computing phase-shift diagrams, often the observations from 2 or $3 \mathrm{yr}$ are combined into one light curve. Thus, if the light curve varies from cycle to cycle, the light curve plotted with observations from more than one year will have scatter due to this. This causes the error in the determination of the points for the $O-C$ diagram.

Wesselink: I have never combined in a mean curve observations which I suspected of showing a change in the form of the light curve. So there was no problem in defining epochs of maximum light for $O-C$ diagrams for period change. 